\title{
ARTICLES \\ EQUILIBRIUM IN A REINSURANCE SYNDICATE; EXISTENCE, UNIQUENESS AND CHARACTERIZATION
}

\author{
By KNut K. Aase \\ Norwegian School of Economics and Business Administration \\ 5035 Bergen-Sandviken, Norway
}

\begin{abstract}
This paper attempts to give an overview of the pricing of risks in a pure exchange economy, where trade takes place at time zero and where uncertainty is revealed at time one. An economic equilibrium model under uncertainty is formulated, where conditions characterizing a Pareto optimal exchange equilibrium are derived. We present two sets of sufficient conditions for the existence of an equilibrium, and demonstrate how equilibria can be characterized through several examples. Uniqueness of equilibrium is also discussed. Special attention is given to the principal components that the premiums in a reinsurance market must depend upon. We also apply the general theory to the risk exchange problem between a policyholder and an insurer, and in particular we compute market premiums of the resulting optimal contracts.

It is emphasized throughout how the formulation of a competitive equilibrium, rather than merely a general risk exchange formulation, is of particular interest in deriving a well-defined and unique set of equilibrium premiums in an insurance market. The theory is put into a framework which is fruitful for extensions beyond the one-period case.
\end{abstract}

\section{KEYWORDS}

Reinsurance market; competitive equilibrium; uniqueness of premiums; Pareto optimality; risk exchange; private insurance; CAPM; risk tolerance; complete markets.

\section{INTRODUCTION}

The following model is interpreted as a reinsurance syndicate, in which I insurers trade among themselves. We take as given

(a) The preference of insurer $i \in I=\{1,2, \ldots, I\}, \succ_{i}$, represented by expected utility $E\left\{u_{i}(\cdot)\right\}$, where $u_{i}^{\prime}>0$ and $u_{i}^{\prime \prime}<0$.

(b) The initial net reserve of insurer $i$, represented by the random variable $x_{i}$, $i \in I$.

We assume that each $x_{i} \in L^{2}(\Omega, \mathscr{F}, P)$ where $(\Omega, \mathscr{F}, P)$ is the probability space on which all the $x_{i}^{\prime}$ 's are defined, all the insurers agree on the probability 
measure $P$ (homogeneous beliefs), and the events $F$ on which $P$ is defined are generated by the I net reserves, which we sometimes call the initial portfolios, i.e., $F=\sigma\left\{x_{1}, x_{2}, \ldots, x_{1}\right\}$. This means that the uncertainty in the model is totally described by the initial portfolios $x_{i}, i \in I$. We briefly comment on the realism of homogeneous beliefs in reinsurance: The assumption about homogeneous beliefs appears reasonable for a reinsurance market, where trade is supposed to take place under conditions of umberrimae fidei, and no information is supposed to be hidden. Our pricing results for a reinsurance market are likely to influence premiums in the market for direct insurance as well. In the direct market the assumption about homogeneous beliefs seems more unrealistic. It is likely that the buyers of insurance have more information about the risk they try to cover, than the insurers. This asymmetric information gives rise to adverse selection. In addition, the buyers can directly or indirectly influence events so that the probability distributions of the risks are altered. This can happen since the insurer is usually unable to monitor the insured, and the phenomenon gives rise to moral hazard. Whereas the problem of morel hazard does not seem important in a reinsurance market, the problem of adverse selection may occur since the ceding company usually has more detailed information about the risks they underwrite than the reinsurers. It may of course be tempting for some direct insurer to sell some "bad risks" in the reinsurance market. In the long run this "practice" is not likely to pay off, since the reinsurance industry makes heavy use of a detailed rating system for insurance companies (i.e., Insurance Solvency International), and there exist penalties for such actions.

The competitive equilibrium (CE) that we shall demonstrate in this model, we claim to be of particular interest in insurance, where its importance has been partly overlooked. Insurers of today seem to be turning to finance markets and their models, often without the understanding of the most basic exchange economy that can be thought of, and which we think is of the utmost importance to general insurance markets; the syndicate described in this paper. The present model has also been a key motivation of much of the financial equilibrium theory which has dominated the literature of financial economics.

The usual formulation in insurance settings has been to derive the front of Pareto optimal (PO) risk exchanges, generally uncountable in number. This does not help to find unique premiums, as there will be one set of prices for each different Pareto optimal point. In order to find a well-defined set of premiums in this model, the budget constraints of the insurers must be employed. A well-posed model will then normally determine a unique set of equilibrium premiums subject to a normalization condition.

The paper is organized as follows: In Section 2 we present the economic model of uncertainty. Here we formulate one set of sufficient conditions, the Inada conditions, for the existence of a unique equilibrium, and we demonstrate some properties of this equilibrium. In Section 3 we demonstrate that the $\mathrm{CE}$ is Pareto optimal, and in Section 4 we present examples of how optimal sharing rules might look like, and what their market values are. Here we introduce a different set of sufficient conditions, called properness, for the 
existence of an equilibrium, which turns out to be satisfied in the examples. We discuss when a syndicated market can restrict attention to proportional treaties, and when non-proportional treaties are needed. In the latter case, we argue that this has nothing to do with the market being "incomplete", as has been suggested in the economic literature. At least this is a definition which we do not find fruitful. In Section 5 we demonstrate some properties of risk tolerances, and in Section 6 we risk adjust the probability measure in the present one-period framework. In Section 7 we present an insurance version of the capital asset pricing model, and in Section 8 we rewrite our results on portfolios to treat insurance premiums directly in this syndicate. In Section 9 we employ the results of Sections 2-4 to the general treatment of the risk exchange between a policyholder and an insurer, and in particular to the computation of market premiums of optimal contracts in such models. We end our exposition in Section 10 with some concluding remarks.

\section{THE ECONOMIC MODEL OF UNCERTAINTY}

In the market the I insurers exchange parts of their initial portfolios among themselves. As a result of these exchanges insurer $i$ obtains a final portfolio, represented by the random variable $y_{i}\left(x_{1}, x_{2}, \ldots, x_{\mathrm{I}}\right)$. Market clearing requires that

$$
\sum_{i \in I} y_{i}=\sum_{i \in I} x_{i}=x_{M}, \quad P \text {-a.s. }
$$

since the insurers only trade among themselves, where $x_{M}$ represents the "market portfolio". If some allocation of risks $\left(y_{1}, y_{2}, \ldots, y_{I}\right)$ satisfy $(2.1)$, it is called feasible. The premium functional we denote by $\pi(\cdot)$. In order to prevent arbitrage possibilities this must be a linear functional on $L^{2}(\Omega, \mathscr{F}, P)$. As an illustration of this point, assume on the contrary that $\pi\left(y_{1}+y_{2}\right)>\pi\left(y_{1}\right)+\pi\left(y_{2}\right)$ for two risks $y_{1}$ and $y_{2}$. Then one agent could insure the bundle $\left(y_{1}+y_{2}\right)$ and reinsure separately $y_{1}$ and $y_{2}$. The cash flow at time zero equals $\left\{\pi\left(y_{1}+y_{2}\right)-\pi\left(y_{1}\right)-\pi\left(y_{2}\right)\right\}>0$. The cash flow at time one equals $-\left(y_{1}+y_{2}\right)+y_{1}+y_{2}=0$. This agent has no obligations at time one, so he has made a riskless profit at time zero. This is a money pump, or a "free lunch", which is inconsistent with an economic equilibrium.

By the Riesz' represenation theorem there exists some function $U^{\prime} \in L^{2}(\Omega, \mathscr{T}, P)$ such that

$$
\pi(x)=E\left\{x U^{\prime}\right\}, \quad \forall x \in L^{2}(\Omega, \mathscr{F}, P) .
$$

Since $F=\sigma\left\{x_{1}, x_{2}, \ldots, x_{l}\right\}$, it follows that $U^{\prime}=U^{\prime}\left(x_{1}, x_{2}, \ldots, x_{l}\right)$ is some Borel-measurable function of $\left(x_{1}, x_{2}, \ldots, x_{I}\right)$ (see e.g. TUCKER (1967), Th.1.1). (So far the prime on $U^{\prime}$ is just a matter of notation. Later we show that under the present conditions $U^{\prime}(\cdot)$ is also a derivative of some function as well.) The statement in (2.2) means, in economic terms, that the market is complete in the following sense (BORCH (1962)): 


\section{Definition 1:}

A market model is complete if it assigns a unique value $\pi(x)$ to an arbitrary random variable $x \in L^{2}(\Omega, \mathscr{F}, P)$.

The economic theory of pricing of contingent claims started with Arrow's paper in (1953). BoRCH (1960-62-68) developed these ideas further, and below we present some of the elements of this theory. Consider the following problem:

$$
\max _{y_{i}(\boldsymbol{x}) \in L^{2}} E\left\{u_{i}\left(y_{i}\left(x_{1}, x_{2}, \ldots, x_{I}\right)\right)\right\}
$$

subject to the budget constraint

$$
\pi\left(y_{i}\right) \leq \pi\left(x_{i}\right), \quad i \in I .
$$

In order to avoid bankruptcy problems we also assume that $y_{i} \geq 0$ a.s. An equilibrium is a collection $\left(\pi ; y_{1}, y_{2}, \ldots, y_{I}\right)$ consisting of a price functional $\pi($. and a feasible allocation $\left(y_{1}, y_{2}, \ldots, y_{I}\right)$ such that for each $i, y_{i}$ solves problem (2.3-4).

The market value of the portfolio cannot increase when exchanges are settled at market prices. The expected utility of the portfolio can however be increased by such exchanges, and this is the very purpose of reinsurance transactions.

The Lagrangian of this problem is

$$
\mathscr{P}\left(y_{i} ; \lambda_{i}\right)=E\left\{u_{i}\left(y_{i}\left(x_{1}, x_{2}, \ldots, x_{I}\right)\right)-\lambda_{i}\left(y_{i}-x_{i}\right) U^{\prime}\right\} \text {. }
$$

For the purpose of the first result below, in addition to the assumptions (a) and (b) we now make the following three assumptions

(c) The derivatives $u_{i}^{\prime}(x)$ satisfy

$$
\lim _{x \rightarrow \infty} u_{i}^{\prime}(x)=0 \text { and } \lim _{x \downarrow 0} u_{i}^{\prime}(x)=+\infty
$$

(d) The functions $x \rightarrow x u_{i}^{\prime}(x)$ are all nondecreasing.

(e) The aggregate market portfolio $x_{M} \in[\delta, \Delta]$ almost surely for finite constants $A>\delta>0$.

The assumption $u_{i}^{\prime}(0+)=+\infty$ guarantees that the constraint $x_{M} \geq 0$ will never be active, called the Inada condition. The condition $u_{i}^{\prime}(x) \rightarrow 0$ as $x \rightarrow \infty$ can be thought of as a saturation effect. We now present a theorem giving sufficient conditions for the existence of a competitive equilibrium. Assumption (d) is sufficient for uniqueness. The theorem also characterizes the equilibrium.

\section{Theorem 1:}

Suppose assumptions (a)-(c) and (e) hold. Then there exists a CE characterized by

$$
u_{i}^{\prime}\left(y_{i}\left(x_{1}, x_{2}, \ldots, x_{l}\right)\right)=\lambda_{i} U^{\prime}\left(x_{1}, x_{2}, \ldots, x_{I}\right), \quad i \in I, P \text {-a.s. }
$$


where $\lambda_{i}$ are positive constants. If in addition (d) holds, then the CE is unique.

Proof: Existence of $\mathrm{CE}$ in infinite dimensional spaces under out conditions are shown by DUFFIE and ZAME (1989). Uniqueness of an interior CE under the additional assumption (d) has been shown by KaratzAs et al. (1988). As for the characterization (2.6), since the Bernoulli utility functions $u_{i}(\cdot)$ are concave, the program (2.3)-(2.4) is concave for each $i$. By the Saddle Point Theorem, if $\left(y_{i}, \lambda_{i}\right)$ is a saddle point of the Lagrangian for this program, $y_{i}(\boldsymbol{x})$ solves the given program for all $i$. Again because of concavity, the conditions (2.6) are the Euler equations of the maximization problem of the Lagrangian in $y(\cdot)$, which in the present situation are necessary and sufficient for the solution of this sub-problem, since the optimal solution happens to be interior by our conditions. Thus the equations (2.6) must hold.

\section{Remarks :}

- Uniqueness means relative to a normalization. In particular this means that if $\lambda=\left(\lambda_{1}, \lambda_{2}, \ldots, \lambda_{I}\right)$ and if $\lambda^{\prime}=\left(\lambda_{1}^{\prime}, \lambda_{2}^{\prime}, \ldots, \lambda_{I}^{\prime}\right)$ are two different vectors corresponding to a unique $\mathrm{CE}$, then there exists a constant $c>0$ such that $\lambda=c \lambda^{\prime}, \quad c \pi(y ; \lambda)=\pi\left(y ; \lambda^{\prime}\right)$ and $y_{i}(\boldsymbol{x} ; \lambda)=y_{i}\left(\boldsymbol{x} ; \lambda^{\prime}\right), \quad$ where $y_{i}(\boldsymbol{x} ; \lambda)$, $i=1,2, \ldots, \mathrm{I}$, are the optimal sharing rules, or equilibrium allocations, corresponding to the vector $\lambda$, and $\pi(y ; \lambda)$ stands for the corresponding pricing rule. Thus equilibrium premiums can be determined only up to a multiplicative constant, since there can always be a re-evaluation of currency; this is not going to afffect, however, the way in which the insurers share the risks among themselves.

- The present economic interpretation of the function $U^{\prime}(x)$ is that it represents the marginal utility of the market as a whole at the "portfolio point" $\boldsymbol{x}$. Another common interpretation, especially in financial economics and in macroeconomics, is that $U^{\prime}(\boldsymbol{x})$ represents the marginal utility function of some representative insurer, or even of some abstract central planner. A final interpretation is also possible; $U^{\prime}(x)$ is the shadow price per unit of $P$-probability when $\boldsymbol{x}(\omega)=\boldsymbol{x}$ (we return to this in Section 3).

- In Section 4 we present a different set of sufficient conditions for the existence of an equilibrium. It turns out that these conditions are inconsistent with the Inada condition (c), but otherwise they appear to be less restrictive. Here we need the above conditions in order to secure an interior optimum.

Some immediate consequences of (2.6) are:

(i) $\frac{\partial U^{\prime}\left(x_{1}, x_{2}, \ldots, x_{i}\right)}{\partial x_{i}}=\frac{1}{\sum_{r \in I} \frac{\lambda_{r}}{u_{r}^{\prime \prime}\left(y_{r}(\boldsymbol{x})\right)}}, \quad i \in I, P$-a.s. 
This follows from differentiating (2.6). Since the right-hand side above does not depend on $i$, it follows directly that

$$
U^{\prime}\left(x_{1}, x_{2}, \ldots, x_{l}\right) \equiv U^{\prime}\left(x_{M}\right), \quad P \text {-a.s. },
$$

so that only changes in the market portfolio $x_{M}$ affects $U^{\prime}$. Similarly

(ii) $\frac{\partial y_{i}\left(x_{1}, x_{2}, \ldots, x_{I}\right)}{\partial x_{j}}=$

$$
\frac{\lambda_{i}}{u_{i}^{\prime \prime}\left(y_{i}(\boldsymbol{x})\right) \sum_{r \in I} \frac{\lambda_{r}}{u_{r}^{\prime \prime}\left(y_{r}(\boldsymbol{x})\right)}}, \quad i, j \in I, P \text {-a.s. }
$$

which again follows from (2.6). We notice that the right-hand side above does not depend upon $j$. This means that the total derivative

$d y_{i}(\boldsymbol{x})=\frac{\partial y_{i}(\boldsymbol{x})}{\partial x_{j}} d x_{M}=\frac{\partial y_{i}(\boldsymbol{x})}{\partial x_{k}} d x_{M}$, for any $j, k \in I$, and for all $i \in I, P$-a.s.,

so that only changes in the aggregate portfolio $x_{M}$ affects the optimal final portfolios $y_{i}$, i.e.

$$
y_{i}\left(x_{1}, x_{2}, \ldots, x_{1}\right)=y_{i}\left(x_{M}\right), \quad i \in I, \quad P \text {-a.s. }
$$

A consequence of this is that the syndicate members can hand in all their initial portfolios to a pool, and let the pool's clerk distribute parts of $x_{M}$ back to the syndicate's members according to the optimal sharing rules $y_{i}\left(x_{M}\right)$.

Here we remark that the consequences (2.7) and (2.8) could alternatively have been derived more directly from the Saddle Point Theorem.

\section{PARETO OPTIMALITY}

If feasible, an allocation $y_{1}, y_{2}, \ldots, y_{1}$ is Pareto optimal if there is no feasible allocation $z_{1}, z_{2}, \ldots, z_{I}$ such that $E\left(u_{i}\left(z_{i}\right)\right) \geq E\left(u_{i}\left(y_{i}\right)\right)$ for all $i$, with strict inequality for at least one $i$. It is now easy to demonstrate that any competitive equilibrium allocation is Pareto optimal. In order to show this, let $\left(U^{\prime}\left(x_{M}\right), y_{1}, \ldots, y_{I}\right)$ denote the CE in Theorem 1 , and suppose $z$ is a feasible allocation which Pareto dominates $\boldsymbol{y}$. Then $E\left(u_{i}\left(z_{i}\right)\right) \geq E\left(u_{i}\left(y_{i}\right)\right)$ for all $i$ with at least one strict inequality, say for insurer $j$. Since $E\left(u_{j}\left(z_{j}\right)\right)>E\left(u_{j}\left(y_{j}\right)\right)$, we know that $\pi\left(z_{j}\right)>\pi\left(y_{j}\right)$. If for some $i$ the quantity $\delta=\pi\left(y_{i}\right)-\pi\left(z_{i}\right)>0$, we could let $y_{i}^{*}=z_{i}+\delta U^{\prime}\left(x_{M}\right) / \pi\left(U^{\prime}\left(x_{M}\right)\right)$, from which $\pi\left(y_{i}^{*}\right)=\pi\left(y_{i}\right)$. But then, since $u_{i}$ is strictly increasing and $U^{\prime}>0$, we would have $E u_{i}\left(y_{i}^{*}\right)>E u_{i}\left(y_{i}\right)$, which is impossible by the definition of an equilibrium. Thus $\pi\left(z_{i}\right) \geq \pi\left(y_{i}\right)$ for all $i$. Using this, we now have the contradiction $\pi\left(\Sigma x_{i}\right) \geq \pi\left(\Sigma z_{i}\right)>\pi\left(\Sigma y_{i}\right)=\pi\left(\Sigma x_{i}\right)$, which proves the result.

When the optimal solution is interior, an alternative construction is the following: It is well known the Pareto optimal sharing rules are found by solving (see e.g. BoRCH (1960-62) or WILSON (1968))

$$
\max _{y_{i}\left(x_{M}\right) \in L^{2}} E\left\{\sum_{i \in J} k_{i} u_{i}\left(y_{i}\left(x_{M}\right)\right)\right\}
$$


such that

$$
\sum_{i \in I} y_{i}\left(x_{M}\right)=\sum_{i \in I} x_{i}=x_{M}, \quad P \text {-a.s. }
$$

where $k_{1}, k_{2}, \ldots, k_{l}$ are arbitrary positive constants. In two dimensions there is a nice graphical illustration of this point. The Lagrangian of problem (3.1) is

$$
\mathscr{Y}\left\{y_{1}, y_{2}, \ldots, y_{I} ; \lambda\left(x_{M}\right)\right\}=E\left\{\sum_{i \in I} k_{i} u_{i}\left(y_{i}\left(x_{M}\right)\right)-\lambda\left(x_{M}\right) \sum_{i \in I}\left(y_{i}-x_{i}\right)\right\} \text {, }
$$

where the Lagrangian multiplier $\lambda(\cdot)$ is now a Borel-measurable function, so that $\lambda=\lambda\left(x_{M}\right)$ is an - measurable random variable.

Theorem 2: (Borch's Theorem)

Under our assumptions (a) and (b) on preferences, the Pareto optimal sharing rules $y_{i}\left(x_{M}\right)$ are characterized by

$$
k_{i} u_{i}^{\prime}\left(y_{i}\left(x_{M}\right)\right)=\lambda\left(x_{M}\right), \quad i \in I, \quad P \text {-a.s. }
$$

where the $k_{i}$ are arbitrary positive constants.

Proof: We assume that $k_{i}$ can be chosen in such a way that the domains of the functions $k_{i} u_{i}^{\prime}(\cdot)$ have a nonvoid intersection. Then there exists at least one Pareto optimal treaty (see DU MoUchel (1968)). By the concavity of the Bernouilli utility functions $u_{i}(\cdot)$, our program is concave. If $\left(y_{i}, \lambda\right)$ is the saddle point of the Lagrangian in (3.2), $y_{i}$ solves the problem (3.1) since $\lambda(\cdot)$ is continuous (this latter property follows since any positive linear functional on $L^{2}$ is continuous). The saddle point must maximize the Lagrangian (3.2) in $y$, and this latter problem can be solved by the calculus of variations: Because of concavity of the $u_{i}$ for all $i$, a necessary and sufficient condition for this maximization is again given by the Euler equations. In this special case they are given by (3.3), since the derivatives of $y_{i}\left(x_{M}\right)$ with respect to $x_{M}$ are not entering the equations (3.2).

\section{Corollary 1:}

The competitive equilibrium of Theoream 1 is Pareto optimal.

Proof: By comparing (3.3) and (2.6-8), the result follows after simply identifying $U^{\prime}\left(x_{M}\right)$ with $\lambda\left(x_{M}\right)$ and $k_{i}$ with $1 / \lambda_{i}$. Alternatively, see the introduction to this section. 
We may notice that the identification in the above proof also explains that the function $U^{\prime}(\cdot)$ may be interpreted as a shadow price, which is exactly what $\lambda(\cdot)$ is. Finally we notice that the formulation (2.3) and (2.4) implies that the CE solutions $y_{i}\left(x_{M}\right), i \in I$, must satisfy individual rationality, since clearly the solution $y_{i}\left(x_{M}\right)=x_{i}$ is possible, where $x_{i}$ is square integrable and obviously satisfies (2.4).

\section{EXISTENCE AND UNIQUENESS OF CE: EXAMPLES}

\subsection{Introduction}

Theorem 1 gives a set of sufficient conditions for a $\mathrm{CE}$ when premiums, as a result of a competitive equilibrium, are unique. Since equilibrium prices can be determined only up to a multiplicative constant, we should normally get unique premiums from the budget constraints after normalization. The family of solutions we get by varying the normalization constant will not affect the sharing rules, as will be demonstrated in the examples below. It turns out, however, that the conditions (c) and (d) for existence and uniqueness are far from necessary. After the examples we shall therefore present an alternative set of sufficient conditions for existence of $\mathrm{CE}$. When the sharing rules are linear, it is possible to reach a Pareto optimum by an exchange of fractions of the initial portfolios. Linear sharing rules are optimal when the individual utility functions are members of the HARA class. In a reinsurance market this means that there should be no need for any other contract than the standard proportional reinsurance contract when this is true. Applied to a stock market, the assumption that the optimal sharing rules are linear implies that there should be no need for trading any other securities than ordinary shares (common stock). Non-proportional reinsurance and securities such as contingent claims and options both exist and are important, so we must conclude that the preferences of decision makers are at least so diverse that they cannot be represented HARA-utility functions only. For some reason many economists refer to a market in which it is impossible to reach a Pareto optimum through an exchange of proportions of the initial portfolio as an "incomplete market".

\subsection{Illustrations}

Example 1: Exponential utility.

Here

$$
u_{i}^{\prime}\left(x_{i}\right)=\exp \left\{-x_{i} / \alpha_{i}\right\}, \quad \alpha_{i}>0, \quad i \in I .
$$

Notice that neither (c) nor (d) hold true here. Nevertheless we shall demonstrate both existence and uniqueness of an interior $\mathrm{CE}$.

Borch's Theorem gives

$$
k_{i} u_{i}^{\prime}\left(y_{i}\left(x_{M}\right)\right)=U^{\prime}\left(x_{M}\right), \quad i \in I, \quad P \text {-a.s. },
$$


which leads directly to

$$
U^{\prime}\left(x_{M}\right)=\exp \left\{\frac{K-x_{M}}{\sum_{i \in I} \alpha_{i}}\right\}, \text { where } K=\sum_{i \in I} \alpha_{i} \ln k_{i} .
$$

Notice how the marginal utility of the market depends upon the parameters $\alpha_{i}$ from the individual preferences, and the positive constants $k_{i}$. The latter can finally be determined from the budget constraints (2.4), which we return to below.

We also notice that the optimal sharing rules are given by

$$
y_{i}\left(x_{M}\right)=\frac{\alpha_{i}}{\Sigma_{j \in I} \alpha_{j}} x_{M}+\left(\alpha_{i} \ln k_{i}-\alpha_{i} \frac{K}{\Sigma_{j \in I} \alpha_{j}}\right), \quad i \in I, \quad P \text {-a.s., }
$$

which verifies that the sharing rules are linear in $x_{M}$. The Arrow-Pratt measure of absolute risk aversion equals $1 / \alpha_{i}$ for each insurer $i$. Also the relative risk aversion is increasing in the net reserves for these insurers. The kind of treaty given in (4.2) seems common in reinsurance pratice. Insurer $i$ will hold a share $\alpha_{i} / \Sigma \alpha_{j}$ of the total market, inversely proportional to his coefficient of absolute risk aversion. In order to compensate for the fact that the least risk-averse insurer will hold the larger proportion of the market, zero-sum side-payments, or fees, occur between the insurers. The last term in (4.2) represents these fees. The quotas are determined by the risk-aversion parameters only. Quota-share treaties with side-payments also occur when all the insurers have preferences represented by logarithmic utilities, quadratic utilities, as well as by power utility functions with the same exponent. For further details see LEMARIE (1990).

Let us for simplicty write (4.2) as

$$
y_{i}\left(x_{M}\right)=\frac{\alpha_{i}}{\Sigma_{j \in I} \alpha_{j}} x_{M}+\beta_{i}
$$

where

$$
\beta_{i}=\left(\alpha_{i} \ln k_{i}-\alpha_{i} \frac{K}{\Sigma_{j \in I} \alpha_{j}}\right)
$$

Employing the budget constraints (2.4), we determine these constants as follows

$$
\beta_{i}=\frac{E\left(x_{i} \exp \left\{-\frac{x_{M}}{A}\right\}-\frac{\alpha_{i}}{A} x_{M} \exp \left\{-\frac{x_{M}}{A}\right\}\right)}{E\left(\exp \left\{-\frac{x_{M}}{A}\right\}\right)}, i=1,2, \ldots, \mathrm{I},
$$


where $A=\Sigma \alpha_{j}$, so that the sharing rules are now uniquely determined. Moreover, the ray $\left(k_{1}, k_{2}, \ldots, k_{I}\right)$ is unique modulo a multiplicative constant. Normalizing so that

$$
K=\sum_{i \in I} \alpha_{i} \ln k_{i}
$$

we obtain the unique ray as follows

$$
k_{i}=\exp \left\{\frac{\beta_{i}}{\alpha_{i}}\right\} e^{\frac{K}{A}}, \quad i=1,2, \ldots, \mathrm{I} .
$$

In the case where we have a riskless security in the economy in addition to the existing portfolios, it is natural to normalize so that $E\left\{U^{\prime}\left(x_{M}\right)\right\}=1$, in which case the normalization constant $K$ is determined from

$$
e^{-\frac{K}{A}}=E\left(\exp \left\{-\frac{x_{M}}{A}\right\}\right)
$$

so that the unique vector of constants $\left(k_{1}, k_{2}, \ldots, k_{I}\right)$ is given by

$$
k_{i}=\frac{\exp \left\{\frac{\beta_{i}}{\alpha_{i}}\right\}}{E\left\{\exp \left(-\frac{x_{M}}{A}\right)\right\}}, \quad i=1,2, \ldots, \mathrm{I} .
$$

Finally the unique set of market premiums of the optimal portfolios $y_{i}$ is given as

$$
\pi\left(y_{i}\left(x_{M}\right)\right)=\frac{\alpha_{i}}{A} \frac{E\left\{x_{M} \exp \left(-x_{M} / A\right)\right\}}{E\left\{\exp \left(-x_{M} / A\right)\right\}}+\beta_{i}=\frac{E\left\{x_{i} \exp \left(-x_{M} / A\right)\right\}}{E\left\{\exp \left(-x_{M} / A\right)\right\}}, \quad i \in I,
$$

i.e., by the Esscher premium principle of actuarial sciences (see BÜHLMANN (1980)).

We now present another example.

Example 2: Logarithmic utility.

Here

$$
u_{i}\left(x_{i}\right)=\ln \left(\beta_{i}+\alpha_{i} x_{i}\right), \quad \text { where } \quad\left(\beta_{i}+\alpha_{i} x_{i}\right)>0 \quad P \text {-a.s., } \quad \alpha_{i}>0 .
$$

The individual marginal utilities are given by

$$
u_{i}^{\prime}\left(x_{i}\right)=\frac{\alpha_{i}}{\beta_{i}+\alpha_{i} x_{i}}, \quad i \in I, \quad P \text {-a.s. }
$$


and the absolute risk aversion and the relative risk aversion are both increasing with net reserve levels if $\beta_{i}>0$. In this case neither condition (c) nor (d) hold. Borch's Theorem gives

$$
\frac{k_{i} \alpha_{i}}{\beta_{i}+\alpha_{i} y_{i}\left(x_{M}\right)}=U^{\prime}\left(x_{M}\right), \quad i \in I, \quad P \text {-a.s. }
$$

which leads to

$$
U^{\prime}\left(x_{M}\right)=\frac{\sum_{i \in I} k_{i}}{\sum_{i \in I} \beta_{i} / \alpha_{i}+x_{M}}, \quad P \text {-a.s. }
$$

and the linear sharing rules

$$
y_{i}\left(x_{M}\right)=\frac{k_{i}}{\sum_{j \in I} k_{j}} x_{M}+\left(\frac{k_{i}}{\sum_{j \in I} k_{j}} \sum_{j \in I} \frac{\beta_{j}}{\alpha_{j}}-\frac{\beta_{i}}{\alpha_{i}}\right), \quad i \in I, \quad P \text {-a.s. }
$$

Using the budget constraints (2.4) we obtain the unique ray $\left(k_{1}, k_{2}, \ldots, k_{I}\right)$ subject to $\Sigma k_{i}=k$, as

$$
k_{i}=k E\left(\frac{x_{i}+\beta_{i} / \alpha_{i}}{A+x_{M}}\right), \quad i=1,2, \ldots, \mathrm{I},
$$

where

$$
A=\sum_{j \in I} \frac{\beta_{j}}{\alpha_{j}}
$$

In the case when the normalization is $E\left\{U^{\prime}\left(x_{M}\right)\right\}=1$, then the constant $k$ equals

$$
k=\left(E\left(\frac{1}{A+x_{M}}\right)\right)^{-1}
$$

so that the unique vector of positive constants $k_{i}$ is given by

$$
k_{i}=E\left(\frac{x_{i}+\beta_{i} / \alpha_{i}}{A+x_{M}}\right)\left(E\left(\frac{1}{A+x_{M}}\right)\right)^{-1}, \quad i=1,2, \ldots, \mathrm{I},
$$

Finally the market values of the optimal portfolios $y_{i}$ are given by

$$
\pi\left(y_{i}\left(x_{M}\right)\right)=E\left\{-\frac{x_{i}}{A+x_{M}}\right\}\left(E\left(\frac{1}{A+x_{M}}\right)\right)^{-1}, \quad i=1,2, \ldots, \mathrm{I},
$$

which is a new "premium principle".

We now present an example where both the conditions (c) and (d) hold true. 
Example 3: Power untility.

Here

$$
u_{i}\left(x_{i}\right)=x_{i}^{p_{i}}, \quad \rho_{i} \in(0,1), \quad i \in I .
$$

If condition (e) hold true, by Theorem 1 there exists a unique CE, which means that this model is complete by our Definition 1. Borch's Theorem gives

$$
k_{i} \rho_{i}\left(y_{i}\left(x_{M}\right)\right)^{\rho_{i}-1}=U^{\prime}\left(x_{M}\right), \quad i \in I,
$$

and the optimal allocations are

$$
y_{i}\left(x_{M}\right)=\left(\frac{1}{k_{i} \rho_{i}} U^{\prime}\left(x_{M}\right)\right)^{1 /\left(\rho_{i}-1\right)}, \quad i \in I,
$$

so that from the market clearing condition we obtain the equation

$$
\sum_{i \in I}\left(\frac{1}{k_{i} \rho_{i}} U^{\prime}\left(x_{M}\right)\right)^{1 /\left(\rho_{i}-1\right)}=x_{M}
$$

The normalization $E\left\{U^{\prime}\left(x_{M}\right)\right\}=1$ together with the budget constraints finally determine the constants $k_{i}$. As an illustration, consider the case where $\mathrm{I}=2$ and $\rho_{1}=1 / 2, \rho_{2}=3 / 4$. Here

$$
y_{1}\left(x_{M}\right)=\left(k_{1} \rho_{1}\right)^{2}\left(U^{\prime}\left(x_{M}\right)\right)^{-2}, \quad y_{2}\left(x_{M}\right)=\left(k_{2} \rho_{2}\right)^{4}\left(U^{\prime}\left(x_{M}\right)\right)^{-4} .
$$

Only the ratio between the two positive constants matter, so we can arbitrarily set $k_{2}=4 / 3$. The marginal utility of the market equals

$$
U^{\prime}\left(x_{M}\right)=\left(\frac{\sqrt{h}+\sqrt{h+4 x_{M}}}{2 x_{M}}\right)^{1 / 2},
$$

and the optimal allocations are

$$
y_{1}\left(x_{M}\right)=\frac{1}{2}\left(\sqrt{h^{2}+4 h x_{M}}-h\right), \quad y_{2}\left(x_{M}\right)=x_{M}+\frac{1}{2}\left(h-\sqrt{h^{2}+4 h x_{M}}\right),
$$

where

$$
h=\left(\frac{\rho_{1}}{\rho_{2}} \frac{k_{1}}{k_{2}}\right)^{4}
$$

The normalization $E\left\{U^{\prime}\left(x_{M}\right)\right\}=1$ finally gives the equation for the constant $h$ (or really $k_{1}$ ), in which case the unique CE is determined. It should be clear that this Pareto optimum can not be achieved by an exchange of proportional reinsurance contracts. Similarly, in a stock market this type of arrangement can not be reached by an exchange of common stock. 
Let us finally check condition (e). Supposing that $x_{M}$ is uniformly distributed on the interval $[\delta, 1+\delta]$ where $\delta$ is some given parameter, we see that here is $\delta=0$ allowed, since the integral $\int_{0+} u^{-1 / 2} d u$ converges. In this particular case (e) is too strong.

\subsection{Uniform properness}

We now turn to another set of sufficient conditions for the existence of an equilibrium.

Following Mas-Colell (1986) and Mas-Colell and Zame (1991), we define an expected utility function $U(y)=E\{u(y)\}$ to be $x$-proper on $X=L_{+}^{2}(\Omega, \mathscr{F}, P)$ (or uniformly proper) if there exists a scalar $\varepsilon>0$ such that for all $y$ in $X, \alpha>0$ and $z$ in $X, U(y-\alpha x+z) \geq U(y)$ implies that $\|z\| \geq \alpha \varepsilon$. Here $\|z\|=\left(E\left\{z^{2}\right\}\right)^{1 / 2}$.

The interpretation is that the portfolio $x$ is desirable, in the sense that loss of an amount $\alpha x$ cannot be compensated for by an additional amount $\alpha z$ for any portfolio $z$, if $z$ is sufficiently "small". When preferences are convex, properness of $U$ at $y$ with respect to $x$ is equivalent to the existence of a premium functional $U^{\prime}$ such that $\pi(z)=E\left\{z U^{\prime}\right\} \geq \pi(y)=E\left\{y U^{\prime}\right\}$ whenever $U(z) \geq U(y)$ and has the additional property that $\pi(x)>0$. The portfolio $x$ in this definition is said to be extremely desirable for $U$. Thus, under risk aversion properness at $x_{M}$ is equivalent to the linear premium rule we know must exist, or individual properness at $x_{M}$ is equivalent to market supportability of $\pi$.

Now, it is known that properness of $U_{i}(y)=E\left\{u_{i}(y)\right\}$ at $x$ is equivalent to the assertion that the random variable $u_{i}^{\prime}(x)$ satisfies $E\left\{\left(u_{i}^{\prime}(x)\right)^{2}\right\}<\infty$. A quasi-equilibrium is defined by the existence of a $U^{\prime} \in X, U^{\prime} \neq 0$, such that $\pi\left(x_{i}\right)=E\left\{x_{i} U^{\prime}\right\}=\pi\left(y_{i}\right)$ and $\pi(v) \geq \pi\left(y_{i}\right)$ whenever $U_{i}(v) \geq U_{i}\left(y_{i}\right)$. A quasiequilibrium is an equilibrium if $U_{i}(v)>U_{i}\left(y_{i}\right)$ implies that $\pi(v)>\pi\left(y_{i}\right)$ for all $i$. This latter property holds at a quasi-equilibrium if $\pi\left(x_{i}\right)>0$ for all $i$. The following result is of interest in our model of an insurance market.

\section{Thereom 3:}

Suppose our conditions (a) and (b) hold and that there is any allocation $z \geq \mathbf{0}$ with $\Sigma z_{i}=x_{M} P$-a.s. If $x_{M}>0 P$-a.s. and $E\left\{\left(u_{i}^{\prime}\left(z_{i}\right)\right)^{2}\right\}<\infty$ for each $i$, then there exists a quasi-equilibrium.

- The proof of this theorem can be adapted from MAS-COLELL and ZAME (1991).

- Consider now the examples above, and suppose the theorem holds for $\boldsymbol{z}=\boldsymbol{y}$, the optimal allocation. The conditions for properness are then

$$
E\left\{\exp \left(-2 y_{i}\left(x_{M}\right) / \alpha_{i}\right)\right\}<\infty, E\left\{\frac{\alpha_{i}^{2}}{\left(\beta_{i}+\alpha_{i} y_{i}\left(x_{M}\right)\right)^{2}}\right\}<\infty, E\left\{y_{i}\left(x_{M}\right)^{2\left(\rho_{i}-1\right)}\right\}<\infty .
$$


for the Examples 1, 2 and 3 respectively. As an illustration, suppose that $x_{M}$ is exponentially distributed with probability density $f(x)=\lambda \exp \{-\lambda x\}$, $x \geq 0, f(x)=0, x<0$. The properness condition in Example 1 is then equivalent to $A=\Sigma \alpha_{i}>0$, which is indeed one of our assumptions. In Examples 2 and 3 the properness requirement does not seem to add any new restrictions to the ones that are already naturally present.

- One advantage with our conditions (a)-(e) is the interior solution they provide, which gives us the characterization (2.6). Theorem 3 does not rule out corner solutions.

- Uniform properness is incompatible with the condition $u_{i}^{\prime}(0+)=+\infty$.

- Uniform properness was used in a model of a reinsurance market in AASE (1990).

\section{RISK TOLERANCE}

Here we demonstrate a simple consequence of Borch's Theorem:

$$
\frac{u_{i}^{\prime \prime}\left(y_{i}\left(x_{M}\right)\right) y_{i}^{\prime}\left(x_{M}\right)}{u_{i}^{\prime}\left(y_{i}\left(x_{M}\right)\right)}=\frac{U^{\prime \prime}\left(x_{M}\right)}{U^{\prime}\left(x_{M}\right)}, \quad i \in I, \quad P \text {-a.s. }
$$

which follows from differentiating (2.6). Equation (5.1) can alternatively be written

$$
\frac{y_{i}^{\prime}\left(x_{M}\right)}{R\left(x_{M}\right)}=\frac{1}{R_{i}\left(y_{i}\left(x_{M}\right)\right)}, \quad i \in I, \quad P \text {-a.s. }
$$

where $R=-\frac{U^{\prime \prime}}{U^{\prime}}$, and $R_{i}=-\frac{u_{i}^{\prime \prime}}{u_{i}^{\prime}}$

stand for absolute risk aversion. Since $\Sigma_{i} y_{i}^{\prime}\left(x_{M}\right)=1$, we see that

$$
\frac{1}{R\left(x_{M}\right)}=\sum_{i \in I} \frac{1}{R_{i}\left(y_{i}\left(x_{M}\right)\right)}, \quad P \text {-a.s. }
$$

The quantity $1 / R$ is called the risk tolerance. The above result has been found by BORCH (1985); see also BüHLMANN (1980) for the special case of exponential utility functions. The result (5.3) says that in a Pareto optimum the risk tolerance of the market as a whole is equal to the sum of the risk tolerances of the participants. If one member is risk neutral, his risk tolerance will be infinite, and hence that of the market. This may be interpreted as saying that in a Pareto optimum all risk should be carried by the risk neutral participants. We can also easily derive the following

$$
\frac{\partial y_{i}\left(x_{M}\right)}{\partial x_{M}}=\frac{R\left(x_{M}\right)}{R_{i}\left(y_{i}\left(x_{M}\right)\right)}, \quad i \in I, \quad P \text {-a.s. }
$$

If all the syndicate's members are strictly risk averse, then $R_{i}>0$, and $R>0$ follows from (5.3), so that $y_{i}^{\prime}\left(x_{M}\right)>0$ a.s. from (5.4). This means that as the 
market portfolio increases, all the insurers increase their portfolios in a Pareto optimum.

\section{RISK ADJUSTMENT OF THE PROBABILITY MEASURE}

The premium functional $\pi$ can alternatively be represented by a risk adjusted probability measure as follows: Suppose there exists a riskless security $x_{0}$ in the economy, and assume without loss of generality that $x_{0}(\omega)=1 P$-a.s. We can then normalize such that $E\left\{U^{\prime}\left(x_{M}\right)\right\}=1$, as we have suggested earlier. Suppose that $P\left[U^{\prime}\left(x_{M}\right)>0\right]=1$. Define a new measure $P^{*}$ as follows:

$$
P^{*}(A)=\int_{A} U^{\prime}\left(x_{M}(\omega)\right) d P(\omega)
$$

Clearly $P^{*}(\Omega)=1$ from our normalization assuption. Also it follows from integration theory that $P^{*}(\cdot)$ is countably additive, confirming that $P^{*}$ is a probability measure. Finally $P^{*}$ and $P$ are mutually absolutely continuous with respect to each other, meaning that if $P(B)=0$ then $P^{*}(B)=0$ and if $P^{*}(A)=0$ then $P(A)=0$ for any $A, B \in \mathcal{F}$. Using $P^{*}$ we can express the premium as follows

$$
\begin{aligned}
\pi(x)=E\left(U^{\prime}\left(x_{M}\right) x\right) & =\int_{\Omega} U^{\prime}\left(x_{M}(\omega)\right) x(\omega) d P(\omega) \\
& =\int_{\Omega} x(\omega) d P^{*}(\omega)=E^{*}(x),
\end{aligned}
$$

where $E^{*}$ refers to the expectation operator under $P^{*}$. The interpretation is that the market premium can be computed using an altered probability measure $P^{*}$ corresponding to a world of market risk neutrality. We call $P^{*}$ the risk adjusted probability measure. Notice from (6.1) that the market's marginal utility $U^{\prime}\left(x_{M}\right)$ corresponds to the Radon-Nikodym derivative of $P^{*}$ with respect to $P$, i.e.

$$
U^{\prime}\left(x_{M}\right)=\frac{d P^{*}}{d P} .
$$

This type of construction is of considerable importance in the timecontinuous case (see e.g., AASE (1988-92-93).

Returning to the illustrations in Section 4.2, we now see that in general the Radon-Nikodym derivative depends on the preferences. This at least holds in equilibrium models. This fact should be contrasted with the literature on contingent claims analysis. In the arbitrage pricing theory, where the uncertainty is modeled by Ito-diffusions, this quantity is preference independent, which clearly does not hold when "jumps" can occur as in our model. 


\section{INSURANCE PREMIUMS}

The foregoing has been formulated in terms of portfolios and market values of net reserves. To obtain market premiums of insurance contracts, we note that the net reserves of insurer $i$ consist of assets $a_{i}$ less of liabilities under the insurance contracts held by the insurer. Let the non-negative random variable $z_{i}(\omega)$ represent claim payments under the contracts if the state of the world becomes $\omega \in \Omega, \quad i \in I$. Let the events be completely specified by $\mathscr{F}=\sigma\left(z_{1}, z_{2}, \ldots, z_{l}\right)$, so that the assets $a_{i}$ are riskless, and write

$$
x_{i}=a_{i}-z_{i}, \quad i \in I \text {. }
$$

Now we have that

$$
\pi\left(x_{i}\right)=a_{i}-\pi\left(z_{i}\right)=a_{i}-E\left\{U^{\prime}\left(a_{M}-z_{M}\right) z_{i}\right\},
$$

where $a_{M}=\Sigma a_{i}$ and $z_{M}=\Sigma z_{i}$. We define the market disutility of claim payments by the function $V$, where

$$
V\left(z_{M}\right)=U^{\prime}\left(a_{M}-z_{M}\right) \text {. }
$$

Clearly $V^{\prime}\left(z_{M}\right)=-U^{\prime \prime}\left(a_{M}-z_{M}\right)>0$ because of assumption (a) and (5.3). Formula (7.2) simply says that the market value of the insurer's portfolio is equal to his riskless assets less the market premium for insurance of the liabilities. This formula makes it easy to translate results expressed in terms of net reserve values into insurance premiums. Notice in particular that if for some portfolio $x_{i}$ the premium $\pi\left(x_{i}\right)<E\left(x_{i}\right)$, we get from (7.2) that the corresponding insurance premium satisfies $\pi\left(z_{i}\right)>E\left(z_{i}\right)$, so that the economic risk premium $\left\{\pi\left(z_{i}\right)-E\left(z_{i}\right)\right\}$ of this insurance contract is positive. After normalization, we find in general that the risk premium can be written as follows

$$
\pi\left(z_{i}\right)-E\left\{z_{i}\right\}=\operatorname{cov}\left\{z_{i}, V\left(z_{M}\right)\right\}, \quad i \in I .
$$

Since the marginal disutility of the market increases as the aggregate claims in the market increase, from (7.4) we may be tempted to believe that for claims which are positively correlated with $z_{M}$, the risk premium is positive, and for claims which are negatively correlated with $z_{M}$, the risk premium is negative. Both these cases make perfectly sense in a rational reinsurance market with risk averse insurers. However, there exist joint distributions for $z=\left(z_{1}, z_{2}, \ldots, z_{I}\right)$ under which this result may not hold true. Covariances are measures of linear statistical dependance, and can only be considered as a good measure of "stochastic association" under multinormality. In insurance an assumption of jointly normally distributed claims is usually not very realistic. Among other things can claims only take on non-negative values. We are therefore reluctant to use the nice results obtainable from an assumption of multinormality in insurance. Here we cite HARALD Cramér (1930) who wrote: “... in many cases the approximation obtained by using the normal function is not sufficiently good to justify the conclusions that have been drawn in this way". In the last section of the paper we nevertheless briefly discuss multinormality. 


\section{RISK EXCHANGE BETWEEN A POLICYHOLDER AND AN INSURER}

The problem of risk exchange between a buyer of insurance and an insurer has been extensively studied under varying conditions in insurance economics, and some of the contributions can also be found in the actuarial literature. By restricting attention to the buyers problem only, Mossin (1968) showed that if the compensation $c(x)=\alpha x$ is received by the policyholder if the damage amounts to $x$, where $0<\alpha<1$ is a constant, and if the premium paid is $\alpha p$, then if $p>E x$ it is never optimal to take full coverage. Borch later modified this, and considered instead a premium $p=\alpha E x+c$, where $c \geq 0$ is some constant. He showed, simply using Jensen's inequality, that $\alpha^{*}=1$ is optimal if it is rational for the risk-averse customer to buy insurance. The constant $c$ he interpreted as administrative costs. ARROW (1974) used Borch's original risk-exchange model of (1960-62), and found that a policy with a deductible is optimal. His premium contains a fixed percentage loading, which has later been interpreted as a special example of a cost function by RAVIV (1979), who analyzed the problem for general cost functions, using the maximum principle. Here we remark that a loading is perhaps more naturally associated with an economic risk premium. HoLMSTRÖM (1979) analyzed the problem under moral hazard, and showed that this gives rise to deductibles. Moral hazard is clearly a problem in this particular kind of risk exchange. ROTHSCHILD and STIGLiTz (1976) considered the case with imperfect information, and demonstrated deductibles for low-risk individuals in a very simple model, and TOWNSEND (1979) established deductibles under a certain kind of nonobservability, where there is a cost involved by verification of the true state. LANDSBERGER and MEILIJSON (1990), on the other hand, explained deductibles in insurance from another perspective, by the use of preferences derived from so called star-shaped utility functions. MofFET (1979) used Borch's Theorem directly on the risk exchange problem that we discuss below.

In this section we want to demonstrate that the risk exchange model of this paper can be used to establish some simple, yet general results, still abstracting from the problems caused by asymmetric information and moral hazard. These results, we claim, constitute the natural benchmark from which refinements should be obtained. In particular we are interested in the form of the premium functional in this situaion, derived from (2.2).

To this end we consider a policyholder with initial wealth $w_{1}$, utility function $u_{1}$ satifying conditions (a) and (b). Against a premium $p$ the insurer offers a policy that reimburses the policyholder an amount $I(x)$ if a claim of amount $x$ occurs. The insurer has initial wealth $w_{0}$, and his utility function we denote by $u_{0}(\cdot)$ satisfying $u_{0}^{\prime}(\cdot)>0, u_{0}^{\prime \prime}(\cdot) \leq 0$. A natural constraint on the compensation function $I(x)$ is

$$
0 \leq I(x) \leq x \text { for all } x .
$$

Ignoring this constraint for the moment, a direct application of Borch's Theorem to the present sharing arrangement gives

$$
u_{0}^{\prime}\left(w_{0}+p-I(x)\right)=\left(k_{1} / k_{0}\right) u_{1}^{\prime}\left(w_{1}-p-x+I(x)\right) \text {. }
$$


Differentiating (8.1) with respect to $x$ leads to

$$
\frac{\partial I(x)}{\partial x}=\frac{\left(k_{1} / k_{0}\right) u_{1}^{\prime \prime}\left(w_{1}-p-x+I(x)\right)}{u_{0}^{\prime \prime}\left(w_{0}+p-I(x)\right)+\left(k_{1} / k_{0}\right) u_{1}^{\prime \prime}\left(w_{1}-p-x+I(x)\right)} .
$$

Using (8.1), we get directly

$$
\frac{\partial I(x)}{\partial x}=\frac{\frac{u_{1}^{\prime \prime}\left(w_{1}-p-x+I(x)\right)}{u_{1}^{\prime}\left(w_{1}-p-x+I(x)\right)}}{\frac{u_{0}^{\prime \prime}\left(w_{0}+p-I(x)\right)}{u_{0}^{\prime}\left(w_{0}+p-I(x)\right)}+\frac{u_{1}^{\prime \prime}\left(w_{1}-p-x-I(x)\right)}{u_{1}^{\prime}\left(w_{1}-p-x+I(x)\right)}},
$$

which can be written

$$
\frac{\partial I(x)}{\partial x}=\frac{R_{1}\left(w_{1}-p-x+I(x)\right)}{R_{0}\left(w_{0}+p-I(x)\right)+R_{1}\left(w_{1}-p-x+I(x)\right)},
$$

where $R_{1}$ and $R_{0}$ again stand for the measures of absolute risk aversion. If both parties are risk averse, then from (8.3) we see that

$$
0<I^{\prime}(x)<1 \text { for all } x \geq 0 .
$$

Letting $I(0)=0$, the mean value theorem implies that

$$
0<I(x)<x \text { for all } x \geq 0 .
$$

This means that the Pareto optimal sharing rule involves a positive amount of coinsurance, or full coverage is not Pareto optimal.

Notice that policies with a deductible can not be Parero optimal. This follows since $I(x)=0, x \leq d, I(x)=x-d, x>d$ has $I^{\prime}(x)=0, x \leq d$ and $I^{\prime}(x)=1, x>d$, both violating (8.4). This holds quite generally without using any constraints on the compensation function $I(x)$.

Referring to the literature cited above, policies with a deductible can only be Pareto optimal in models where one or more of the following are included; costs, moral hazard, asymmetric information, non-observability or alternative preferences (e.g., star-shaped utility).

Example 1: (Exponential utility).

Suppose $u_{1}\left(w_{1}\right)=1-\exp \left\{-a w_{1}\right\}, u_{0}\left(w_{0}\right)=1-\exp \left\{-b w_{0}\right\}$ for two positive constants $a$ and $b$. In this case $R_{0}=b$ and $R_{1}=a$, so the absolute risk aversions are constants and independent of wealth levels. It now follows directly from (8.3) that $I^{\prime}(x)=a /(a+b)$, or $I(x)=a x /(a+b)+c$, where $c$ is an intergration constant. If $I(0)=0, c=0$. In this case if $R_{1}=a$ is large compared to $R_{0}=b, I(x)$ is approximately equal to $x$, so that full coverage is then approximately Pareto optimal. In practice this seems reasonable, since the absolute risk aversion of the policyholder is usually large compared to that of the insurer. 
From (8.3) we see that the conclusion of this example also holds quite generally, i.e., if $R_{1}$ is very large as compared to $R_{0}$ for all input values, then full coverage is approximately Pareto optimal. Quite generally, if we tried to solve the risk exchange problem in this section imposing the natural constraints on $I(x)$, the application of the maximum principle would yield the same conclusions as above: The Pareto optimal deductible is zero in the absence of operating expenses (RAviv (1979)).

Turning to the premium, the problem of determining $p$ is usually overlooked or ignored in the above kind of analyses, where $p$ is simply assumed to be given as "a positive number".

Suppose we use the pricing principles of Section 2 of this paper, and apply them to the present "mini-market". We would like to answer the question of how the resulting equilibrium-based premium $p$ depends on the parameters of the problem. First we need to derive the shadow price. Using Borch's Theorem we get

$$
k_{1} u_{1}^{\prime}\left(w_{1}-p-x+I(x)\right)=U^{\prime}\left(w_{1}+w_{0}-x\right)
$$

and

$$
k_{0} u_{0}^{\prime}\left(w_{0}+p-I(x)\right)=U^{\prime}\left(w_{1}+w_{0}-x\right) .
$$

The budget constraints of the two parties are

$$
\pi\left\{y_{0}\left(x_{M}\right)\right\}=\pi\left\{x_{0}\right\}
$$

and

$$
\pi\left\{y_{1}\left(x_{M}\right)\right\}=\pi\left\{x_{1}\right\} .
$$

Here $y_{0}\left(x_{M}\right)=w_{0}+p-I(x), y_{1}\left(x_{M}\right)=w_{1}-p-x+I(x), x_{M}=w_{1}+w_{0}-x=$ $w-x, x_{0}=w_{0}$ and $x_{1}=w_{1}-x$. Using (8.8) we have $E\left\{\left(w_{0}+p-I(x)\right) U^{\prime}\left(x_{M}\right)\right\}$ $=E\left\{w_{0} U^{\prime}\left(x_{M}\right)\right\}$, and since

$$
p=\pi\{I(x)\}=E\left\{I(x) U^{\prime}\left(x_{M}\right)\right\},
$$

we obtain that the by now familiar normalization $E\left\{U^{\prime}\left(x_{M}\right)\right\}=1$ must hold. Consider the following example:

Example 2: (Exponential utility, continued).

Using the results of the above example and of Example 1 in Section 4, we get the following: The shadow price equals $U^{\prime}(w-x)=\exp \{(K-w+x) / A\}$ where $K=\left(\ln k_{1}\right) / a+\left(\ln k_{0}\right) / b$ and $A=1 / a+1 / b$. From the normalization we find the constant $K=w-A \ln \{E[\exp (x / A)]\}$. Furthermore, from Example 1 we get, in the case where $I(0)=0$, that the market premium $p$ is given by

$$
p=E\left(I(x) U^{\prime}\left(x_{M}\right)\right)=\frac{a}{a+b} \cdot \frac{E\{x \exp (x / A)\}}{E\{\exp (x / A)\}} .
$$

As an illustration, suppose that $x$ is exponentially distributed with parameter $\lambda$, so that $\mathrm{Ex}=1 / \lambda$. Then the simple formula $p=a /[\lambda(a+b)-a b]$ obtains, 
where the parameter $\lambda>1 / A$. Notice that $p$ increases with $E x$ and with $a$. If $\lambda<a$, then $p$ increases with $b$, with the opposite result if $\lambda>a$. Notice that an increase in $R_{1}=a$ has here two effects, both working in the same direction: First the absolute risk aversion of the policyholder increases, and second the coverage increases; so we would expect a large premium $p$ in both cases. An increase in $R_{0}=b$ implies on the one hand less coverage, but on the other hand the insurer becomes more averse towards risk. These two mutually competing facts explain the more complex comparative statics for $b$.

The risk neutral case can be studied by letting $b \rightarrow 0$. Then $p \rightarrow E x$ follows from (8.11) by the monotone convergence theorem, i.e., we obtain the usual "actuarial fair" premium in the limit. Alternatively we could try the characterization in Section 2 directly with $u_{0}(w)=b+c w, c>0$, a constant. It is then straightforward to show that the shadow price $U^{\prime}\left(x_{M}\right) \equiv 1$, again leading to premium given by the "principle of equivalence" above. (Formally the latter derivation is not valid when $u_{0}^{\prime \prime}(w)=0$ for all $w$.)

Examples 1 and 2 are somewhat specialized in that the absolute risk aversion is independent of wealth. In general we should also expect the premium to depend on the aggregate level of wealth $w$ in the market. This is indeed of importance in actual markets where insurance contracts are traded at market prices. Consider the following example:

Example 3: (Power utility).

Suppose $u_{0}(w)=u_{1}(w)=w^{\rho}$ where $\rho \in(0,1)$. In this case the shadow price is

$$
U^{\prime}\left(x_{M}\right)=\frac{(w-x)^{\rho-1}}{\left(\frac{1}{\left(\rho k_{0}\right)^{1 /(\rho-1)}}+\frac{1}{\left(\rho k_{1}\right)^{1 /(\rho-1)}}\right)},
$$

which becomes, after the standard normalization

$$
U^{\prime}\left(x_{M}\right)=\frac{(w-x)^{\rho-1}}{E\left\{(w-x)^{\rho-1}\right\}} .
$$

The Pareto optimal sharing rule satisfies

$$
\frac{\partial I(x)}{\partial x}=\frac{w_{0}+p-I(x)}{w-x},
$$

depending, as we see, on the premium $p$. Solving this differential equation under the condition $I(0)=0$ gives

$$
I(x)=\frac{w_{0}+p}{w} x,
$$

i.e., full coverage is only Pareto optimal if $p=w_{1}$. The present problem is 
well-posed for the above utility functions only if $x \leq \min \left(w_{0}, w_{1}\right) P$-a.s. Since $p<w_{1}$ must generally hold, coinsurance results. The premium $p$ must satisfy $p=E\left\{x U^{\prime}\left(x_{M}\right)\left(w_{0}+p\right) / w\right\}$, which leads to

$$
p=\left(\frac{w}{E\left\{x U^{\prime}\left(x_{M}\right)\right\}}-1\right)^{-1} w_{0} .
$$

Notice how the premium $p$ in general depends on the wealth level $w$. It is seen that unless the wealth of the customer is too large, i.e., when $w_{1}<E\left\{x U^{\prime}\left(x_{M}\right)\right\}$, the premium decreases as $w_{0}$ increases as well as when $w_{1}$ increases, whereas the premium increases as a function of $w_{0}$ when $w_{1}>E\left\{x U^{\prime}\left(x_{M}\right)\right\}$. In general the premium is a decreasing function of $w$. This is in accordance with the general observation that the premiums tend to decrease as the "capacity" $(=w)$ in the market increases and vice verca.

In the limiting case where $\rho \rightarrow 1, U^{\prime}\left(x_{M}\right) \rightarrow 1$ a.s. and $p \rightarrow w_{0} E x /(w-E x)$ by the dominated convergence theorem. In the limit, approaching risk neutrality, the optimal compensation scheme is $I(x)=w_{0} x /(w-E x)$, costing its actuarial fair value $E(I(x)\}=w_{0} E x /(w-E x)$.

As an illustration, suppose that $x$ is uniformly distributed on $\left(0, w_{1}\right)$, where $w_{0}>w_{1}$. The premium is then

$$
p_{\rho}=\frac{w_{0}\left[w\left(w^{\rho}-w_{0}^{\rho}\right) / \rho-\left(w^{\rho+1}-w_{0}^{\rho+1}\right) /(\rho+1)\right]}{\left(w^{\rho+1}-w_{0}^{\rho+1}\right) /(\rho+1)},
$$

which depends on the aggregate wealth $w$ of the two parties, their attitude towards risk as measured by $\rho$ and the reserves $w_{0}$ of the insurer. As $\rho \rightarrow 1$, this expression is seen to converge to $p_{1}=w_{1} w_{0} / 2\left(w-w_{1} / 2\right)$, which is exactly $E\left\{I_{1}(x)\right\}$, where $I_{1}(x)=\left(w_{0}+p_{1}\right) x / w$ is the optimal sharing rule for this particular premium $p_{1}$.

\section{AN INSURANCE VERSION OF THE CAPITAL ASSET PRICING MODEL}

We now discuss the case when $\boldsymbol{x}=\left(x_{1}, x_{2}, \ldots, x_{I}\right)$ is jointly multinormally distributed. As noted before, this case has limited applicability in insurance economics. However some of the results in this model remain true even if the assumption of normality is dropped. The first problem we encounter is to find a set of sufficient conditions for the existence of a competitive equilibrium. Our earlier theorems can not be directly applied here, since $\boldsymbol{x}$ can take on negative values with positive probability. NIELSEN (1990) has a set of sufficient conditions for the existence of equilibrium in a CAPM-model in financial economics. In his model the investor has a utility function $U(\mu, \sigma)$ which is a function of the mean and the standard deviation of the total portfolio return. Mean-variance behavior is consistent with expected utility maximization with general utility functions if the returns follow the distributions described by Chamberlain (1983) and OWEN and RABINOWITCH (1983), which include the multinormal distribution. In the present model the optimal allocation may not 
be a linear function of $x_{M}$, in which case $y$ is not necessarily multinormally distributed. Expected utility maximization with general utility functions can not, in our model of an insurance market, in general be represented by mean-variance utility functions $U(\mu, \sigma)$, unless the utility functions happen to be members of the HARA-family with the same cautiousness. In this latter case Nielsen's sufficient conditions are possibly appropriate in our model.

For the moment supposing an interior equilibrium exists, its characterization is then straightforward. Under our normalization assumption $E\left\{U^{\prime}\left(x_{M}\right)\right\}=1$, the premium functional can be written

$$
\pi\left(x_{i}\right)=E\left(x_{i}\right)+\operatorname{cov}\left(x_{i}, U^{\prime}\left(x_{M}\right)\right) \text { for all the } x_{i} .
$$

Here $\left(\pi\left(x_{i}\right)-E\left(x_{i}\right)\right)$ is the economic risk premium of $x_{i}$. From the assumption of multinormality it follows that

$$
\operatorname{cov}\left(x_{i}, U^{\prime}\left(x_{M}\right)\right)=E\left\{U^{\prime \prime}\left(x_{M}\right)\right\} \operatorname{cov}\left(x_{i}, x_{M}\right) \text { for all the } x_{i} \text {. }
$$

Since (9.2) holds for each of the initial portfolios, clearly

$$
\pi\left(x_{i}\right)=E\left(x_{i}\right)+E\left\{U^{\prime \prime}\left(x_{M}\right)\right\} \operatorname{cov}\left(x_{i}, x_{M}\right) \text { for all the } x_{i} .
$$

By summation over $i$ we obtain

$$
\pi\left(x_{M}\right)=E\left(x_{M}\right)+E\left\{U^{\prime \prime}\left(x_{M}\right)\right\} \operatorname{var}\left(x_{M}\right)
$$

from the linearity of the pricing functional $\pi(\cdot)$ and from standard properties of the expectation and the covariance operators. Rearranging, we finally have the insurance version of the capital asset pricing model as follows:

$$
\pi\left(x_{i}\right)-E\left(x_{i}\right)=\frac{\operatorname{cov}\left(x_{i}, x_{M}\right)}{\operatorname{var}\left(x_{M}\right)}\left(\pi\left(x_{M}\right)-E\left(x_{M}\right)\right), \text { for all } i
$$

The risk premium of any of the initial portfolios can be written as the risk premium of the market portfolio multiplied by the normalized covariance term, the portfolio's beta in the market.

The result (9.2) is often referred to as Stein's lemma. The first derivation in the economics literature seems to be due to RUBinSTEIN (1973). Using a Taylor series expansion, he assumed that the function $U^{\prime}$ possesses derivatives of all orders and that these functions can be integrated. Below we give a simple proof, where $U^{\prime}$ need not even be one time differentiable for (9.3) to result by the above procedure.

\section{Lemma 1:}

Suppose $(X, Y)$ is jointly bivariate normally distributed. Then

$$
\operatorname{cov}(X, g(Y))=\frac{\operatorname{cov}(Y, g(Y))}{\operatorname{var} Y} \operatorname{cov}(X, Y) .
$$


Suppose $g^{\prime}(\cdot)$ exists for all real numbers and that $E\left|g^{\prime}(Y)\right|<\infty$. Then

$$
E\left(g^{\prime}(Y)\right)=\frac{\operatorname{cov}(Y, g(Y))}{\operatorname{var} Y} .
$$

Proof: From the assumption of binormality it follows that $E(X \mid Y)=\alpha+\beta Y$, where

$$
\beta=\frac{\operatorname{cov}(X, Y)}{\operatorname{var} Y} \text { and } \alpha=E X-\beta E Y .
$$

Also cov $(X, g(Y))=E\{E\{X g(Y) \mid Y\}\}-E X E g(Y)=\alpha E g(Y)+\beta E(Y g(Y))$ $-\operatorname{EXEg}(Y)=\beta\{E(Y g(Y))-E Y E g(Y)\}$, proving (a). As for (b), by integration by parts, using the assumption that the expectation of $g^{\prime}(Y)$ exists, we find

$$
E g^{\prime}(Y)=-\int_{-\infty}^{\infty} g(y) f_{Y}^{\prime}(y) d y=\frac{1}{\operatorname{var} Y} E\{g(Y)(Y-E Y)\}
$$

where $f_{Y}(y)$ is the normal probability density function for $Y$. This proves (b).

Note that (9.3) follows from (a) only. Thus the assumptions that $U^{\prime}$ is one time differentiable and that the expectation of $U^{\prime}\left(x_{M}\right)$ exists, are really not needed in the above step. If $U^{\prime \prime}$ exists for all reals together with its expectation, then (a) and (b) imply (9.2). For an extension of this result, proved by entirely different methods, see WEI and LEE (1988).

Note that we have used the equilibrium-result in Section 2 that $U^{\prime}(\boldsymbol{x})=U^{\prime}\left(x_{M}\right)$.

We may also find the sign of the risk premium of any Pareto optimal, linear sharing rule $y_{i}\left(x_{M}\right)$. In this case we find

$$
\pi\left(y_{i}\left(x_{M}\right)\right)-E\left(y_{i}\left(x_{M}\right)\right)=E\left(\frac{\partial y_{i}\left(x_{M}\right)}{\partial x}\right)\left(\pi\left(x_{M}\right)-E\left(x_{M}\right)\right), \text { for all } i
$$

By (5.4) we notice that this beta is positive. The risk premium of all the portfolios have then the same sign as the risk premium of the market portfolio, which in this case is negative. This result corresponds to "investors hold efficient portfolios in capital market equilibrium" in the theory of capital markets, whereas the fact that $E\left\{y_{i}^{\prime}\left(x_{M}\right)\right\}>0$ corresponds to "efficient portfolios have positive betas". Notice that the negative risk premiums here only mean that the insurers require a positive expected return on their reinsurance exchanges, since this expected return simply equals $\left[E\left(y_{i}\right)-\pi\left(y_{i}\right)\right] / \pi\left(x_{i}\right)>0$.

Returning to (9.3) suppose that one of the initial portfolios, $x_{1}$ say, has a negative correlation with the market. The market finds this portfolio so valuable that it accepts a negative expected return on $x_{1}$ in equilibrium. 
As for the characterization (9.3), there might exist other joint probability distributions giving the same separation result (see for example Ross (1976) and Chamber lain (1983)). A different line of attack has traditionally been to impose further conditions on preferences. For example, if the marginal utility of the market $U^{\prime}$ is linear, then this separation follows as well.

Apparently this result seems to require no assumptions regarding the joint probability distribution of $\boldsymbol{x}$. However, linear marginal market utility is usually a consequence of quadratic utility functions representing the preferences of the individual insurers, which means that the probabilities of falling beyond the satiation points should equal zero in order for condition (a) to remain valid. Otherwise the preferences are not monotonic, and risky investments are inferior compared to the riskless. Thus conditions must then indirectly be imposed on the joint probability distribution of $\boldsymbol{x}$ as well. For example is the multinormal distribution not acceptable in this situation. In such cases the conditions of Theorem 1 may be met, and the characterization (9.3) be valid. We should add, however, that one obvious advantage of imposing distributional assumptions on $\boldsymbol{x}$ rather than assumptions directly on preferences (if these can at all be avoided), is that the former can be empirically tested using statistical methods, whereas the latter are much harder to verify/refute from available data.

The classical version of the one-period CAPM in a capital market was developed by Sharpe, Lintner and Mossin. The classical one-period CAPM has also been developed without the assumption of a riskless asset by BLACK (1972). In a multiperiod setting MERTon (1972) has developed an intertemporal CAPM, where the prices of the risky assets are assumed to follow Ito-diffusions. In a dynamic, intertemporal reinsurance context, where the claims processes are represented by random, marked point processes, an insurance version of an intertemporal CAPM can be found in AASE (1993).

\section{SUMMARY}

From the above analysis we observe that the premiums in a reinsurance market typically must depend on:

(i) The stochastic properties of the risk itself.

(ii) The stochastic relationship between the particular risk $z$ and claims in the market as a whole, described by the covariance between $V\left(z_{M}\right)$ and $z$.

(iii) The attitude towards risk in the market as a whole, represented by $V=U^{\prime}$.

(iv) The total assets of all the insurers in the market, represented by $a_{M}$.

A realistic theory of insurance premiums must of course take all these four elements into account. This is however rarely done in actuarial risk theory. Several books have been written on insurance premium principles, some even recent, where only the first of these four elements are covered.

Some obvious weaknesses of the above model are the following. There is in reality no time dimension in these models; trade is supposed to take place only at one point in time, and the world more or less ends at the next time point. In 
models of (re)insurance markets the risks may be more realistically represented by random, marked point processes. A model where trade can take place at any time point $t$ in an interval $[0, T]$ is given in AASE (1992-93). There it is shown that the market's attitude towards risk can be separated into two components; one related to frequency risk and the other related to claim size risk, given that an accident has occurred. In order to fully understand these results, however, it appears to be essential to have the above model in mind. This is so since the present derivation basically tells us what happens at each time point of jump of the vector $\boldsymbol{x}$ of the stochastic process representing the exogenously given portfolios in the reinsurance market. For example is our interpretation of the market marginal utility crucial also in the dynamic case. Therefore the one-period analysis can be viewed as a necessary preparation in order to proceed to more realistic, but at the same time more complicated and mathematically challenging models of equilibrium premium formation in a dynamic exchange economy under uncertainty.

\section{ACKNOWLEDGEMENT}

I would like to thank LARS TYGE NiELSEN for stimulating discussions on some topics of this paper, and also an anonymous referee for discovering several errors and misprints.

\section{REFERENCES}

Aase, Knut K. (1993) Premiums in a Dynamic Model of a Reinsurance Market. Scandinavian Actuarial Journal (to appear).

Aase, Knut K. (1992) Dynamic Equilibrium and the structure of Premiums in a Reinsurance Market. The Geneva Papers on Risk and Insurance Theory 17:2, 93-136.

AASE, KNUT K. (1990) Stochastic equilibrium and premiums in insurance. In: Approche Actuarielle des Risques Financiers, $I^{\text {er }}$ Colloque International AFIR,-Paris 59-79.

AASE, KNUT K. (1988) Contingent claims valuation when the security price is a combination of an Ito process and a random point process. Stochastic Processes and their Applications 28, $185-220$.

ARrow, K.J. (1953) Le rôle des valeurs boursiers pour la répartition la meilleure des risques. Colloques Internationaux du CNRS, XL, Paris 41-88.

ARrow, K. J. (1974) Optimal insurance and generalized deductibles. Skandinavisk Aktuarietidsskrift, $1-42$.

BLACK, F. (1972) Capital market equilibrium with restricted borrowing. Journal of Business 45, $444-454$.

BORCH, K.H. (1960) The safety loading of reinsurance premiums. Skandinavisk Aktuarietidsskrift, $163-184$.

BORCH, K.H. (1962) Equilibrium in a reinsurance market. Econometrica 30, 424444.

Borch, K. H. (1967) The theory of risk. Journ. of the Royal Statist. Soc., B 29, 432-452.

BorCH, K.H. (1968) General equilibrium in the economics of uncertainty. In: Risk and Uncertainty, (Editors: Karl Henrik Borch and Jan Mossin), London, Macmillan.

Borch, K. H. (1985) A theory of insurance premiums. The Geneva Papers on Risk and Insurance 10, 192-208.

BorCH, K. H. (1990) Economics of Insurance. Advanced Textbooks in Economics Series. (Editors: Knut K. Aase and Agnar Sandmo), North Holland; Amsterdam, New York, Oxford, Tokyo.

Bühlmann, H. (1980) An economic premium principle. ASTIN Bulletin 11, 52-60. 
BühlmanN, H. (1984) The general economic premium principle. ASTIN Bulletin 14, 13-21.

BülmanN, H. and Jewell, W.S. (1979) Optimal risk exchanges. ASTIN Bulletin 10; 3, 249.

Chamberlain, G. (1983) A characterization of the distributions that imply mean-variance utility functions. Journal of Economic Theory 29, 185-201.

CRAMER, H. (1930) On the mathematical theory of risk. Skandia Jubilee Volume. Stockholm.

Duffie, D. (1991) The theory of value in security markets. In: Handbook of Mathematical Economics vol. IV, 1615-1682. (Editors: W. Hildenbrand and H. SonnesChein), North Holland; Amsterdam, New York, Oxford, Tokyo.

DufFIE, D. and ZAME, W. (1989) The consumption-based capital asset pricing model. Econometrica 57, $1279-1297$.

Dreze, J.H. (1987) Essays on economic decisions under uncertainty. Cambridge University Presss; Cambridge, New York.

Du Mouchel, W. (1968) The Pareto-optimality on an $n$-company reinsurance treaty. Skandinavisk Aktuarietidsskrift 51, 165-170.

HaRT, O.D. (1974) On the existence of equilibrium in a securities model. J. Econ. Theory 9 , $293-311$.

Henriet, D. et J.C. Rouchet (1991). Microeconomie de l'assurance, Economics, Paris.

Holmström, B. (1979) Moral hazard and observability. Bell Journal of Economics 10, 74-91.

Karatzas I. (1989) Optimization problems in the theory of continuous trading. SIAM J. Control and Optimization 27; 6, 1221-1269.

Karatzas, I. Lehoczky, J.P. and Shreve S. E. (1990) Existence and uniqueness of multi-agent equilibrium in a stochastic, dynamic consumption/investment model. Mathematics of Operations Research 15; 1, 80-128.

Landsberger, M. and Meilijson, I. (1990) Lotteries, insurance, and star-shaped utility functions. Journal of Economic Theory 52, 1-17.

Lemarie J. (1990) Borch's Theorem: A historical survey of applications. In : Risk, Information and Insurance. Essays in the Memory of Karl H. Borch (Editor: H. LoUBERGÉ). Kluwer Academic Publishers; Boston, Dordrecht, London.

LiEnHARD, M. (1986) Calculation of price equilibria for utility functions of the HARA class. ASTIN Bulletin 16, 91-97.

LintNer, J. (1965) The valuation of risk assets and the selection of risky investments in stock portfolios and capital budgets. Review of Economics and Statistics 47, 13-37.

Mas-Colell, A. (1986) The price equilibrium existence problem in topologicai vector lattices. Econometrica 54, 1039-1054.

Mas-Colell, A. and ZAme, W. R. (1991) Equilibrium theory in infinite dimensional spaces. In: Handbook of Mathematical Economics vol. IV, 1835-1898. (Editors: W. HiLdenBRAND and H. Sonnenschein), North Holland; Amsterdam, New York, Oxford, Tokyo.

Merton, R.C. (1973) An intertemporal capital asset pricing model. Econometrica 41, 867-887.

Moffet, D. (1979) The risk sharing problem. The Geneva Papers on Risk and Insurance 11, $5-13$.

Mossin J. (1966) Equilibrium in a capital asset market. Econometrica 34, $768 \cdots 783$.

Mossin J. (1968) Aspects of rational insurance purchasing. Journal of Political Economy 76, $553-568$.

Nielsen, L.T. (1990) Existence of Equilibrium in CAPM. Journal of Economic Theory 52, $223-231$.

Owen, J. and RABINOvitch, R. (1983) On the class of elliptical distributions and their applications to the theory of portfolio choice. $J$. Finance $38,745-752$.

Raviv, A. (1979) The design of an optimal insurance policy. American Economic Review 69. $84-96$.

Ross, S.A. (1976) The arbitrage theory of capital asset pricing. Journal of Economic Theory, $341-360$.

Rothschild, M. and STIGlitz, J. (1976) Equilibrium in competitive insurance markets. An essay on the economics of imperfect information. Quarterly Journal of Economics 90, 629-650.

Rubinstein, M.E. (1973) A comparative static analysis of risk premiums. Journal of Business 46 , $605-615$.

Sharpe, W.F. (1964) Capital asset prices. A theory of market equilibrium under conditions of risk. Journal of Finance 19, 425-442.

TownsEND, R. (1979) Optimal contracts and competitive markets with costly state verification. $J$. Econ. Theory 21, $265-293$. 
TuCker, H.G. (1967) A graduate course in probability. Academic Press, New York and London. WEI, K.C.J. and LEE, C.F. (1988) The generalized Stein/Rubinstein covariance formula and its application to estimate real systematic risk. Management Science 34, 1266-1270.

WILSON, R. (1968) The theory of syndicates. Econometrica 36, 119-131.

WYLER, E. (1990) Pareto optimal risk exchanges and a system of differential equations: A duality theorem. ASTIN Bulletin 20, 23-32.

KnUt K. Aase

Norwegian School of Economics and Business Administration

5035 Bergen-Sandviken, Norway. 